\title{
Avaliação da percepção de segurança em relação à presença de três tipos de infraestrutura cicloviária em cruzamentos não semaforizados
}

\author{
Claudinei Moreira Diniz ${ }^{1}$, Marcos Antônio Garcia Ferreira ${ }^{2}$
}

${ }^{1}$ Programa de Pós-graduação em Engenharia Urbana, Universidade Federal de São Carlos, claudinei_diniz@hotmail.com 2Programa de Pós-graduação em Engenharia Urbana, Universidade Federal de São Carlos, dmag@ufscar.br

\section{Recebido:}

26 de fevereiro de 2019

Aceito para publicação:

27 de junho de 2019

Publicado:

30 de abril de 2020

Editor de área:

Flávio Cunto

\section{Palavras-chaves:}

Infraestrutura cicloviária,

Segurança viária,

Percepção de segurança.

\section{Keywords:}

Cycling infrastructure,

Road safety,

Safety perception.

DOI:10.14295/transportes.v28i1.1968

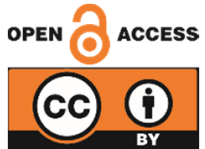

\begin{abstract}
RESUMO
Nos cruzamentos, a concentração de pontos de conflito e a necessidade de circulação em meio ao fluxo de veículos motorizados causa nos ciclistas uma maior sensação de insegurança. Desse modo, o presente estudo analisa a percepção de segurança viária em cruzamentos urbanos não semaforizados em relação a presença de infraestrutura cicloviária dedicada aos ciclistas. A metodologia foi baseada na coleta de dados por questionário on-line que buscou identificar a percepção de segurança de usuários de bicicleta para uma série de cenários, com e sem a presença de infraestrutura cicloviária. Os dados foram analisados por meio de métodos de estatística descritiva e inferencial, nos quais foram comparadas as respostas por tipo de infraestrutura e por tipo conflito. Os resultados encontrados mostram que cenários com a presença de ciclovia no canteiro central tendem a ser percebidos como os mais seguros, enquanto que cenários com vias compartilhadas são percebidos como os mais inseguros.
\end{abstract}

\begin{abstract}
At intersections, the concentration of traffic conflicts and riding amidst motorized vehicles cause a sensation of insecurity in the cyclists. For that matter, several authors cite the implementation of cycling infrastructure as a determining factor to promote bicycle users' safety perception. Thus, this study aims to investigate safety perception at nonsignalized urban intersections in terms of presence of cycling infrastructure. The methodology applied was based on the application of an online survey assessing cyclist's perception in a set of intersection scenarios with cycling infrastructure, all based in Brazilian scenarios. Data were analyzed by methods of inferential statistics, in which were compared safety perception by type of cycling infrastructure. Results show that intersections with cycling track in the median are perceived as the safest, while intersections with shared lanes as the most unsafe.
\end{abstract}

\section{INTRODUCÃO}

Atualmente, cerca de 1,3 milhão de pessoas morrem por ano em decorrência de acidentes de trânsito, número esse que representa a nona maior causa de mortes no mundo (WHO, 2018). No Brasil, a violência do trânsito também vem atingindo valores alarmantes; segundo dados levantados por Waiselfisz (2013), entre os anos 2000 e 2011 ocorreu um aumento de 43,2\% no número de mortes em vias públicas por decorrência de acidentes de trânsito. Um dos fatores apontados como responsável por esse aumento é a expansão da frota de veículos ocorrida no país nas últimas décadas (IPEA, 2015).

Em meio a essa expansão da frota de veículos motorizados estão os ciclistas que, apesar de fazerem parte desse número de mortes no trânsito cada vez mais exorbitante, nem sempre são 
representados em relatórios oficiais em decorrência de uma recorrente subnotificação de acidentes envolvendo as bicicletas. Além disso, a segurança cicloviária nem sempre é considerada nas fases iniciais de elaboração de projetos viários que, por vezes, resulta em um sistema de tráfego perigoso para os ciclistas (Wegman et al., 2012).

Os ciclistas são um dos usuários mais frágeis do sistema de trânsito e, em geral, respondem pela parcela que sofre as lesões mais graves em acidentes envolvendo modos motorizados e as bicicletas (Wei e Lovegrove, 2013; Manton et al., 2016). De acordo com Elvik (2009), os ciclistas possuem uma probabilidade de até 7,5 vezes maior de sofrerem lesões graves em acidentes quando comparados a ocupantes de automóveis.

Nos cruzamentos, o maior número de conflitos de tráfego faz dos problemas de segurança viária enfrentados pelos ciclistas ainda mais evidente. Nessa discussão, diversos estudos já apontaram que os cruzamentos apresentam uma concentração de pontos de conflitos de tráfego que aumentam a probabilidade da ocorrência de acidentes entre bicicletas e automóveis (Wachtel e Lewiston, 1994; Rasanem e Summala, 1998; Wang e Nihan, 2004; Isaksson-Hellman, 2012).

Dessa forma, é importante a compreensão do papel da percepção ao risco de acidentes em cruzamentos urbanos e sua relação com a presença de infraestrutura cicloviária. No contexto apresentado, o presente estudo tem como objetivo geral comparar a percepção de segurança cicloviária em cruzamentos urbanos em termos da presença de três tipos de infraestrutura cicloviária - compartilhamento de vias, vias com ciclofaixa, e vias com ciclovia no canteiro central.

Para alcançar esse objetivo geral, fez-se necessário os seguintes objetivos secundários: desenvolver um instrumento de coleta de dados capaz de analisar a percepção dos ciclistas frente à segurança cicloviária nos cruzamentos; analisar a percepção do ciclista sobre a seguridade oferecida pela infraestrutura viária presente nos cruzamentos urbanos; e identificar fatores positivos e negativos relacionados à infraestrutura oferecida aos ciclistas frente aos conflitos enfrentados em cruzamentos urbanos não semaforizados.

\section{Referencial teórico}

\subsection{Principais Tipos de Infraestrutura Cicloviária no Meio Urbano}

A infraestrutura cicloviária em vias urbanas tem a capacidade de estimular o uso da bicicleta como meio de transporte e, quanto maior a sua presença nas cidades, maior tende ser a taxa de utilização das bicicletas (Menghini et al., 2010; Kaplan e Prato, 2013; Rosenberg, 2015).

Segundo Welle et al. (2016), elementos de infraestrutura cicloviária funcionam como peçaschave para a segurança e promoção da sensação de conforto para os ciclistas. Entretanto, a escolha do tipo de elemento de infraestrutura a ser implementado exige planejamento e cautela pela existência de diferenças nas preferências e perfis de ciclistas em uma determinada região (Heinen; Van Wee; Maat, 2010). Dessa forma, novos elementos de infraestrutura cicloviária podem não resultar em melhorias de segurança para os ciclistas quando não consideradas as características regionais de seus usuários, em especial daqueles que irão utilizar essas novas infraestruturas.

Para o presente estudo, os elementos de infraestrutura cicloviária foram divididos em dois tipos principais: (1) infraestrutura nos cruzamentos e (2) infraestrutura nas aproximações ou saídas de cruzamentos. 
A infraestrutura cicloviária dentro dos cruzamentos é a aquela presente na área de conflito e funciona como um tratamento do ambiente construído para tornar as interseções mais seguras para os ciclistas, bem como para os demais usuários do trânsito. A necessidade desses elementos se faz pela existência de um maior número de conflitos e acidentes nos cruzamentos urbanos (Wang e Nihan, 2004; Isaksson-Hellman, 2012; Nordback; Marshall; Janson, 2014; Denver Public Works, 2016).

Os tipos de tratamentos de interseções são diversos, podendo ser destacados para este estudo a implementação de marcações no pavimento ao longo de cruzamentos, os sistemas de restrição de manobras de conversão e a criação de ilhas de refúgio (Weigand, 2008; Brasil, 2010; NACTO, 2014). Denver Public Works (2016) cita, também, os tratamentos focados no melhoramento do campo de visão do usuário, tais como: eliminação de vagas de estacionamento próximas aos cruzamentos, redução do raio de curvatura de esquinas, inclusão de faixas exclusivas para conversão à direita, pinturas e marcações no piso em regiões de aglomeração de pontos de conflitos e ampliação da infraestrutura cicloviária por toda a extensão dos cruzamentos.

A infraestrutura nas regiões de aproximação e saída de cruzamentos foi dividida em três tipos principais: via compartilhada, ciclofaixa, e ciclovia no canteiro central. A principal diferença entre esses três tipos de infraestrutura é o nível de segregação em relação ao fluxo de veículos motorizados.

As vias compartilhadas são aquelas em que os ciclistas circulam entre os carros e dividem com os demais usuários do sistema de trânsito o mesmo espaço nas faixas de rolamento. Em geral, são implementadas onde o sistema viário já é adequado para esse compartilhamento em função da velocidade de circulação permitida e do volume de tráfego e, por isso, não são necessárias modificações na infraestrutura para integrar a bicicleta ao fluxo de veículos motorizados (Brasil, 2010).

Quando bem implementadas, as vias compartilhadas criam um ambiente mais seguro para a circulação de bicicletas. Em um estudo desenvolvido por Minikel (2012), na cidade de Berkeley, nos Estados Unidos, foi identificado uma taxa de acidentalidade menor em vias compartilhadas quando comparadas com vias arteriais paralelas em uma mesma região. Entretanto, diversos autores citam que a experiência de circular em vias compartilhadas pode causar sensação de insegurança e altos níveis de estresse para os ciclistas menos experientes (Kaplan e Prato, 2013; O'Connor e Brown, 2010).

A ciclofaixa é uma faixa de limitação do espaço dentro de uma via dedicada exclusivamente para a circulação das bicicletas. Essa delimitação é realizada por meio de faixas pintadas no piso, em geral junto ao bordo da pista externa, e por sinalização de trânsito que oferecem à presença dos ciclistas nas pistas um maior destaque e os separam do fluxo imediato de automóveis.

Em relação a esse tipo de infraestrutura, Elvik (2009) observou uma redução estatisticamente significativa dos acidentes com feridos em vias que possuem ciclofaixas implantadas. Lusk et al. (2011) e Teschke et al. (2012) corroboram esse resultado afirmando que as ciclofaixas contribuem para redução do risco de acidentes entre bicicletas e automóveis no sistema de trânsito.

A ciclovia consiste em espaço destinado exclusivamente aos ciclistas e é totalmente segregada do tráfego motorizado. Nas ciclovias, a interação e integração direta com o fluxo de veículos ocorre somente nos cruzamentos. Sua implementação normalmente ocorre ao longo de rios, 
praias, canais, ferrovias (em especial quando desativadas), campi universitários e parques (Brasil, 2010).

A segregação oferecida pelas ciclovias foi identificada como importante em termos de percepção de segurança para os ciclistas avaliados pelo estudo de Chataway et al. (2014), no qual foram coletadas opiniões entre ciclistas de duas cidades, Brisbane, na Austrália, e Copenhagen, na Dinamarca. Entretanto, EMBARQ (2015), destaca que a ciclovia é um elemento desfavorável à segurança viária nos cruzamentos, pois força uma reintegração dos ciclistas ao fluxo dos modos motorizados, o que nem sempre é desejado ou esperado pelos ciclistas.

\subsection{Segurança Cicloviária nos Cruzamentos}

Acidentes e conflitos graves entre bicicletas e veículos motorizados tendem a ocorrer com maior frequência, e em maior número, nas interseções e suas aproximações (Wachtel e Lewiston, 1994; Wang e Nihan, 2004; Isaksson-Hellman, 2012). 0 maior número de ocorrências de conflitos pode ser explicado pela complexidade apresentada pelas interseções no âmbito da engenharia de transportes e, em muitos casos, porque projetos viários nem sempre adotam medidas de inclusão do modo bicicleta de uma forma segura e funcional (Landis et al., 2003).

Além disso, é importante destacar que os ciclistas, diferentemente da maioria dos demais usuários do sistema de trânsito, ao enfrentar situações de conflito de tráfego e de risco imediato também precisam lidar com o equilíbrio e a estabilidade lateral da bicicleta (Ferraz et al., 2012). Dessa forma, a interação dos ciclistas com conflitos de tráfego é uma tarefa que demanda um controle físico e psicológico extra pela necessidade de evitar quedas ao lidar com os riscos de acidentes e com os demais usuários das vias.

Nesse contexto, ressalta-se que compreender a percepção da segurança viária sob a ótica dos ciclistas é importante para a determinação e implementação de projetos de infraestrutura que promovam a segurança em vias urbanas e, consequentemente, incentivam o uso das bicicletas como meio de transporte (Ng et al., 2017).

Entre os estudos abordando a percepção de segurança viária de ciclistas nos últimos anos, Chaurand e Delhomme (2013) avaliaram a percepção de segurança para uma série de situações corriqueiras de conflitos no trânsito, principalmente as interações envolvendo ciclistas e motoristas. No estudo, os autores consideraram a percepção de segurança sob a ótica tanto dos motoristas como a dos ciclistas. Um dos resultados encontrados por eles indica que a interação direta com automóveis tende a provocar uma maior sensação de risco para os ciclistas.

$\mathrm{Ng}$ et al. (2017) investigaram o tipo de infraestrutura cicloviária considerado como o mais seguro em interseções urbanas não semaforizadas na cidade de Queensland, Austrália. Os autores identificaram que ciclistas tendem a preferir infraestruturas que concedem o direito de passagem aos carros durante a execução de manobras de conversão, pois nesses cenários os ciclistas conseguem definir o melhor momento, e o mais seguro, ao passar por uma interseção.

A experiência do ciclista é um fator importante na determinação da sensação de segurança percebida frente a um tipo de infraestrutura. Entretanto, Wachtel e Lewiston (1994), identificaram que ciclistas mais experientes se envolvem mais em acidentes devido a uma maior expectativa em relação ao direito de passagem na interação com veículos motorizados, e também devido a uma maior expectativa de que motoristas não iriam realizar manobras irregulares ou ilegais. 
Outro fator encontrado com recorrência por estudos foi o aumento da percepção de risco em relação ao controle geral na ocorrência de uma situação de conflito. A percepção ao risco tende a ser maior em situações onde é baixo a previsão e controle dos eventos de risco a serem enfrentados, tais como os encontrados em rotatórias e cruzamentos, nos quais é difícil determinar as rotas exatas que serão tomadas pelos veículos (Moller e Hels, 2008; Chaurand e Delhomme, 2013; Ng et al., 2017).

\section{METODOLOGIA}

A metodologia deste trabalho foi desenvolvida com as etapas apresentadas na Figura 1 e descritas nos itens que seguem. Os dados utilizados foram coletados por uma pesquisa de mestrado sendo desenvolvida dentro do Programa de Pós-Graduação em Engenharia Urbana da UFSCar - Universidade Federal de São Carlos.

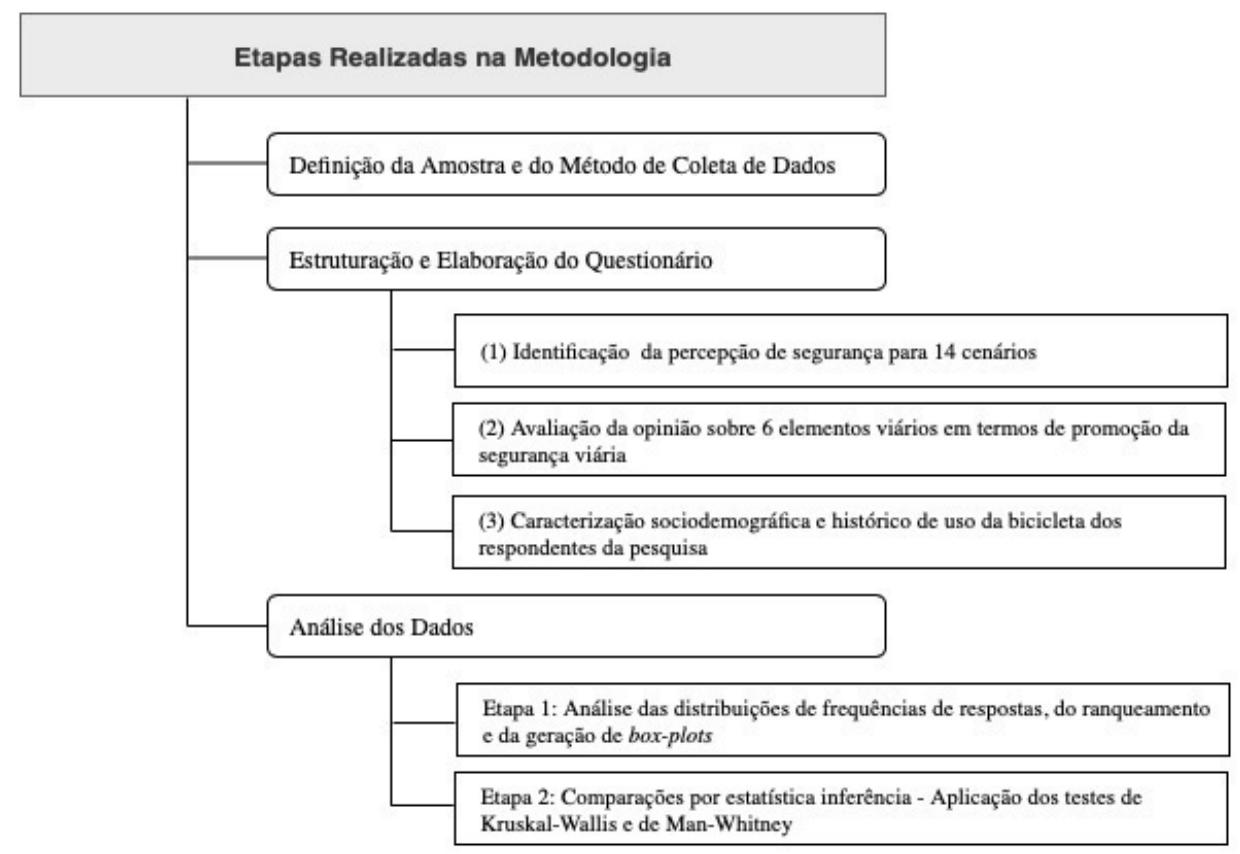

Figura 1. Fluxograma com as etapas da metodologia utilizada

\subsection{Definição da Amostra e do Método de Coleta de Dados}

A amostra utilizada neste estudo foi composta por ciclistas que se autodeclaram usuários de bicicleta como meio de transporte e demais indivíduos que, apesar de não se autodeclararem como ciclistas, possuem alguma experiência de uso da bicicleta no meio urbano.

A coleta de dados foi realizada por meio de aplicação de questionário on-line desenvolvido na plataforma Google Forms e esteve aberto para coleta de respostas nos meses de setembro e outubro de 2018. 0 contato com os respondentes foi realizado por meio de listas de e-mails e por publicações em redes sociais dedicadas a comunidades e associações de ciclistas. Além disso, foi utilizada a estratégia de recrutamento por bola de neve em que todos os respondentes eram convidados a indicar mais participantes para a pesquisa através do compartilhamento do link do questionário. 


\subsection{Estruturação e Elaboração do Questionário}

0 questionário foi estruturado em 3 blocos, que são: (1) identificação da percepção de segurança nas principais configurações e conflitos encontrados em cruzamentos urbanos não semaforizados, (2) avaliação de elementos viários que promovem segurança cicloviária, e (3) caracterização sociodemográfica e histórico de uso de bicicleta.

(a) Conflito de direção oposta com conversão à esquerda

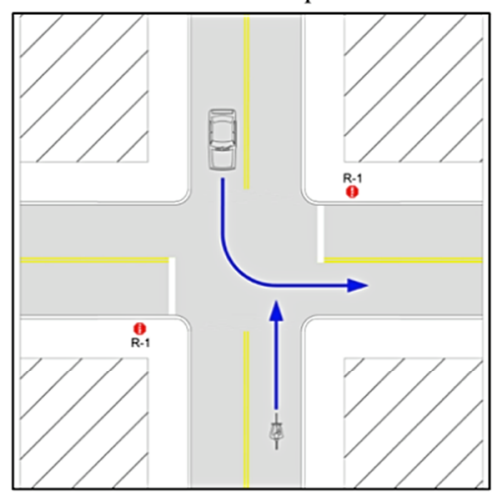

(c) Conflito de conversão à direita com aproximação pela direita

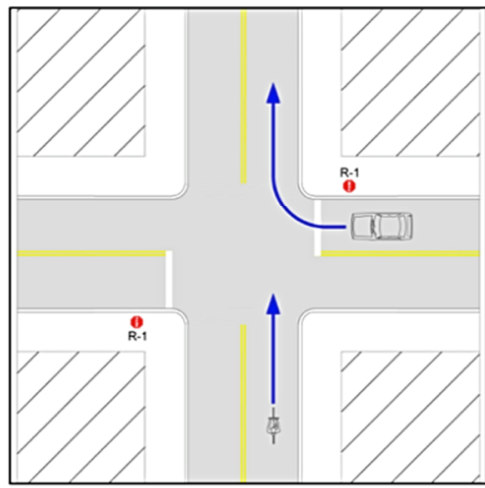

(e) Conflito de mesma direção com conversão à direita

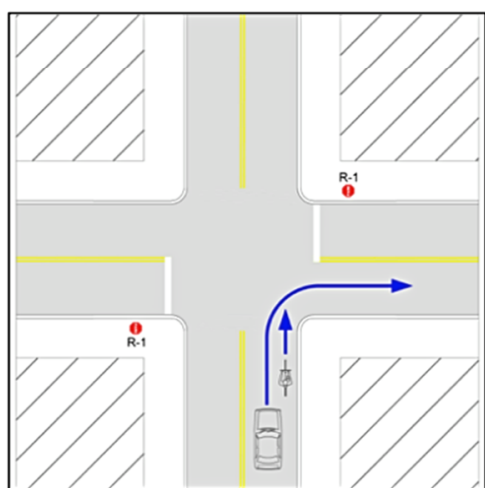

(b) Conflito de cruzamento com aproximação pela esquerda

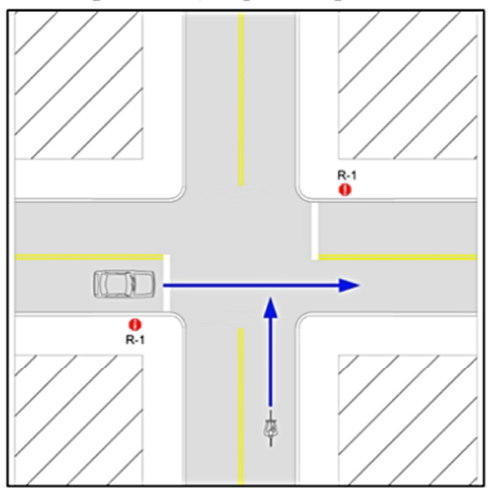

(d) Conflito de conversão à esquerda com aproximação pela direita

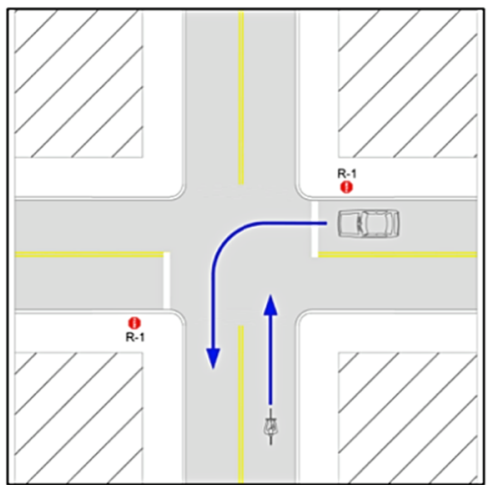

Figura 2. Esquematização da classificação dos conflitos estudados. 
No bloco 1 foram avaliados 14 cenários que apresentavam situações de conflito de tráfego entre automóveis e bicicletas em cruzamentos urbanos não semaforizados. Esses cenários eram representados no formato de imagens em 2D que compreendiam um cruzamento entre uma via secundária e uma via principal com um dos três tipos de infraestrutura cicloviária adotadas neste estudo: via compartilhada, via com ciclofaixa e via com ciclovia no canteiro central. Nesses cenários eram apresentados, ainda, situações de interação entre ciclistas e automóveis em um dos cinco tipos de conflitos apresentados na Figura 2. As esquematizações desses conflitos foram adaptadas da classificação proposta pelo órgão americano Federal Highway Administration - FHWA (2006).

Para cada uma das 14 imagens foi solicitado que o entrevistado classificasse os cenários, considerando a percepção de segurança, em uma escala do tipo Likert variando de 1 a 5, em que 1 indicava que o cenário era "muito inseguro" e 5 "muito seguro". Um exemplo de como a questão era apresentada ao respondente pode ser observado na Figura 3, no qual está sendo avaliado a percepção do ciclista para um cenário com conflito de mesma direção com conversão à direita em um cruzamento que contém ciclofaixa na via principal.

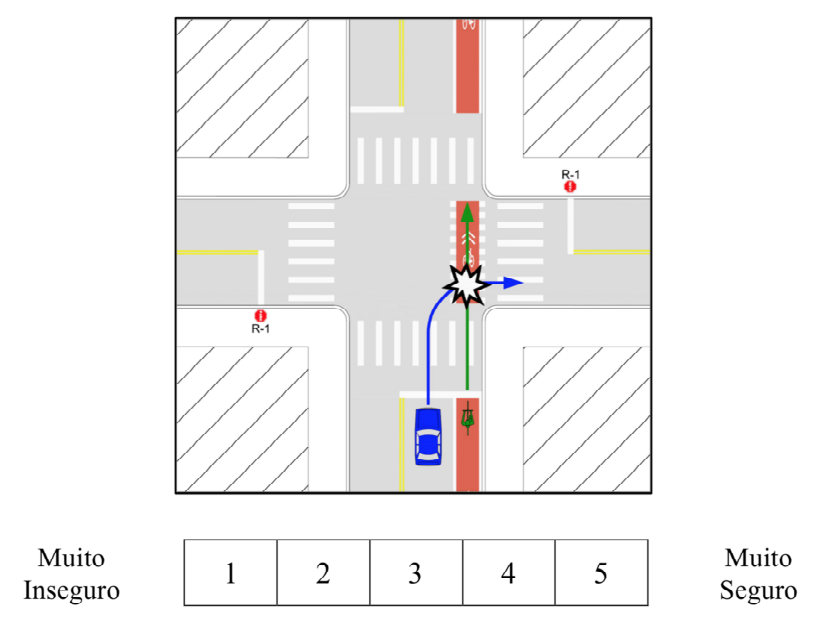

Figura 3. Exemplo de configuração das perguntas para o bloco 1.

O bloco 2 continha sentenças sobre a percepção da melhora da sensação de segurança nos cruzamentos em relação aos seguintes elementos viários: visibilidade em termos de ver e ser visto, largura das faixas de rolamento, iluminação do cruzamento, velocidade dos automóveis, marcações e pinturas no pavimento, e presença de estacionamento nos bordos laterais das vias de aproximação. No total foram avaliadas 6 sentenças, cada uma contendo um dos elementos viários avaliados pelo estudo e o entrevistado poderia classificar sua concordância com a frase em uma escala de 5 pontos do tipo Likert. A escala variava de 1 a 5 , em que 1 representava discordância total com a sentença e 5 concordância total.

No último bloco de perguntas (bloco 3), os participantes responderam questões referentes às suas características sociodemográficas e histórico de uso de bicicleta. Essas perguntas coletavam a cidade em que o respondente costuma andar de bicicleta, gênero, faixa etária, escolaridade, posse de carteira de habilitação, experiência como motorista de automóvel, tipo de usuário de bicicleta (utilitário, recreacional e não ciclista), frequência de utilização de bicicleta e se o respondente já se envolveu em acidentes ou conflitos graves de trânsito. 


\subsection{Análise dos Dados}

A análise dos dados foi realizada em duas etapas e, em ambas, o foco principal foi comparar a percepção de segurança dos cenários avaliados em relação à presença de infraestrutura cicloviária. Os testes foram realizados com auxílio dos softwares RStudio versão 1.1.453 e Excel com a extensão Real Statistics.

A primeira etapa consistiu em comparar as respostas por tipo de infraestrutura por meio da distribuição das frequências de respostas e do ranqueamento pelas medianas, modas e médias das respostas coletadas. Nessa fase, também foram realizadas comparações de cada um dos cinco tipos de conflitos apresentados na Figura 2 em relação aos 3 tipos de infraestrutura (compartilhada, ciclofaixa e ciclovia). Essas comparações permitiram uma melhor visualização das percepções para a presença de infraestrutura cicloviária e foram realizadas por meio da plotagem de gráficos do tipo box-plot.

Com os gráficos do tipo box-plot foi possível apresentar visualmente a distribuição dos dados da amostra de repostas coletadas, tais como a dispersão, simetria, caudas e outliers. A linha (limite) superior da caixa representa o terceiro quartil, a linha (limite) inferior o primeiro quartil, e a linha central a mediana da amostra. A reta vertical, tracejada, é referente a variabilidade da amostra e se estende do limite inferior até o limite superior dos dados. Os pontos nos extremos representam os outliers.

A segunda etapa consistiu da aplicação dos testes de Kruskal-Wallis e o de Mann-Whitney para comparar se as diferenças observadas entre as respostas são estatisticamente significativas. Esses dois testes foram escolhidos porque os dados analisados não apresentaram distribuição normal, fazendo-se necessário a aplicação de testes não-paramétricos.

0 teste de Kruskal-Wallis foi aplicado para comparar as respostas de um mesmo conflito com os 3 tipos de infraestrutura. Nesse teste, buscou-se identificar se um mesmo conflito era percebido de forma diferente nos três tipos de infraestrutura. Em seguida, como teste post-hoc foi aplicado o teste de Mann-Whitney para cada cenário avaliado pelo estudo em par com todos os demais conflitos, formando assim uma tabela cruzada de resultados para todos os 14 cenários estudados.

\section{RESULTADOS E DISCUSSÕES}

\subsection{Perfil dos Respondentes}

Foram coletadas 458 respostas de residentes de diversas cidades brasileiras localizadas nas cinco regiões geográficas. A maioria dos respondentes reside em cidades de grande porte (acima de 500 mil habitantes), nessa categoria destacam-se as cidades São Paulo - SP e Niterói - RJ que apresentaram o maior número de respondentes, com um total de 51 e 30 respostas, respectivamente. Além dessas duas cidades, a cidade de São Carlos - SP, que se enquadra na categoria de cidade de médio porte, também apresentou um maior volume de respostas, com um total de 50 respondentes.

No que se refere ao perfil dos respondentes, foi coletado um número ligeiramente maior de respostas de usuários de bicicleta do sexo masculino $(58,7 \%)$. A faixa etária com maior frequência de respondentes foi a de "25 a 34 anos" $(35,1 \%)$. A escolaridade da maioria dos respondentes foi "Ensino Superior Completo" (72,3\%), número esse que se justifica com a maioria dos respondentes com idade superior ou igual a 25 anos, faixa etária coincidente com a idade média de finalização de todos os níveis de escolaridade. 
Em relação ao tipo de usuário de bicicleta, a maioria dos respondentes declarou ser ciclista do tipo recreacional $(43,4 \%)$, seguido de um número significativo de respondentes que se autodeclararam do tipo utilitário (37,8\%). A maioria dos respondentes $(84,7 \%$ e $66,4 \%$, respectivamente) declarou possuir Carteira Nacional de Habilitação (CNH) e dirigir automóvel frequentemente.

\subsection{Avaliação da Percepção de Segurança por Tipo de Infraestrutura \\ 4.2.1. Comparação das Distribuições de Frequências de Respostas}

Para simplificação das análises e para melhor entendimento da distribuição das opiniões dos respondentes, as 5 categorias de percepção de segurança foram reajustadas para 3 categorias: inseguro (composta pelas categorias muito inseguro e inseguro), indiferente e seguro (composta pelas categorias muito seguro e seguro).

Nas respostas obtidas para os cenários com cruzamentos entre vias compartilhadas, os respondentes tenderam classificar todos os cinco tipos de interações de conflito como inseguro. Com exceção do conflito de conversão à direita e aproximação pela direita (Figura 2 - c), em que o motorista falha em dar prioridade ao ciclista em um sinal de parada obrigatória com aproximação pela direita, todos os conflitos apresentaram mais de 50\% das respostas na categoria que classifica os cenários como inseguro.

Nos cruzamentos entre vias com a presença de ciclofaixa foi observado uma maior distribuição das respostas, principalmente quando comparado com as respostas observadas para os cruzamentos entre vias compartilhadas. Para os cruzamentos entre vias com ciclofaixa, a maioria dos respondentes classificou os cenários como indiferente, o que significa que não são capazes de avaliar o cenário como sendo seguro ou inseguro. Entretanto, observou-se que a porcentagem de distribuição de respostas para cada uma das 3 categorias de percepção de segurança variou entre $30 \%$ e $40 \%$, mostrando um maior balanceamento da distribuição das repostas entre essas categorias.

Nos cruzamentos entre vias com a presença de ciclovia no canteiro central, a maioria dos respondentes classificou os cenários de conflitos como sendo seguro. No entanto, ocorreu uma exceção com o grupo de ciclistas do tipo utilitário. A maioria dos respondentes desse grupo classificou o conflito de mesma direção com conversão à direita (Figura 2 - e) como sendo inseguro. Esse conflito ocorre quando o automóvel se aproxima por trás do ciclista para realizar uma manobra de conversão à direita no cruzamento, o que faz com que essa aproximação do veículo seja de difícil percepção para o ciclista e, consequentemente, pode causar maior sensação de perigo.

Ressalta-se que o conflito de mesma direção e com conversão à direita, discutido anteriormente, também foi o que apresentou as maiores frequências de respostas na categoria inseguro, tanto nos cruzamentos entre vias compartilhadas como para os cruzamentos entre vias com a presença de ciclofaixa na via principal.

Em seguida, foram gerados gráficos do tipo box-plot por tipos de conflitos em que a infraestrutura (compartilhada, ciclofaixa e ciclovia) era avaliada em um mesmo gráfico. Diferentemente do reajuste realizado para a comparação da distribuição de respostas, nos gráficos boxplot as análises foram realizadas com base nas cinco categorias de classificação inicial: muito inseguro, inseguro, indiferente, seguro e muito seguro. Os gráficos gerados podem ser observados na Figura 4. 
Comparando os três tipos de infraestrutura, é possível observar que todo os cincos tipos de conflitos tendem a ser classificados como mais inseguro quando em cruzamentos entre vias com infraestrutura compartilhada. Além disso, percebe-se que para todos os cinco tipos de conflito (Figura 4 - a, b, c, d e e) os cruzamentos entre vias com infraestrutura compartilhada apresentaram o terceiro quartil no valor de 3, resultado esse que indica que pelo menos $75 \%$ de todas as respostas para essa infraestrutura varia entre 1 e 3 (muito inseguro e indiferente).

(a) Conflito de direção oposta com conversão à esquerda

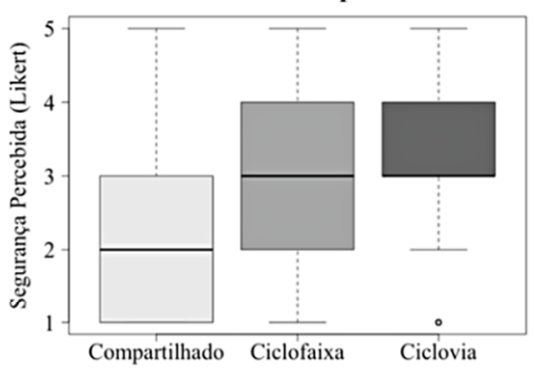

(c) Conflito de conversão à direita com aproximação pela direita

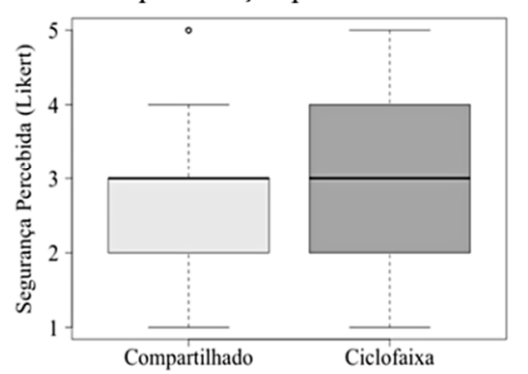

(e) Conflito de mesma direção com conversão à direita

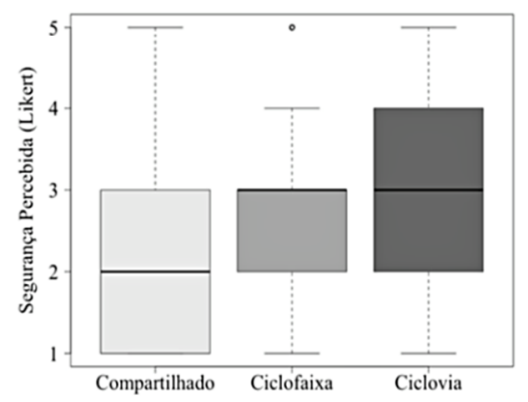

(b) Conflito de cruzamento com aproximação pela esquerda

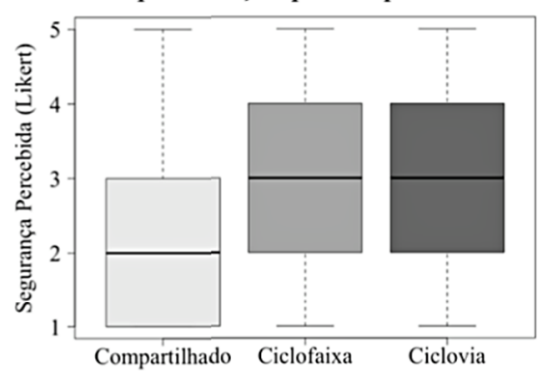

(d) Conflito de conversão à esquerda com aproximação pela direita

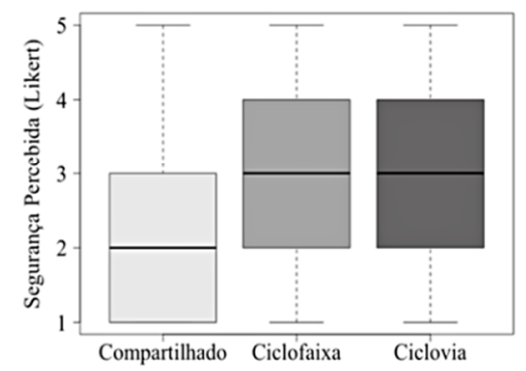

Figura 3. Exemplo de configuração das perguntas para o bloco 1.

Esse primeiro resultado corrobora os estudos de Kaplan e Prato (2013) e O'Connor e Brown (2010), nos quais foi identificado que infraestruturas compartilhadas são percebidas como mais inseguras pelo alto nível de estresse causado pela circulação entre meios de transporte motorizados, em especial para usuários de bicicleta menos experientes. 
Por outro lado, é possível observar uma maior confiança por parte dos respondentes de que os cruzamentos entre vias com a presença de ciclovia no canteiro central oferecem uma maior sensação de segurança. Na Figura 4, quando analisados todos os cinco tipos de conflitos, os cruzamentos entre vias com ciclovia no canteiro central apresentaram respostas com os limites dos quartis em categorias superiores (mais seguras). Essas respostas em categorias mais seguras ficam ainda mais evidentes quando são comparadas com os outros dois tipos de infraestruturas estudadas (vias compartilhadas e vias com ciclofaixa).

Nesses resultados, destacam-se os cenários com o conflito de direção oposta com conversão à esquerda que apresentaram o primeiro quartil no valor de 3 para a infraestrutura ciclovia (Figura $4-\mathrm{a}$ ), indicando que pelo menos $75 \%$ das respostas foram avaliadas entre as categorias de indiferente e muito seguro.

Tal resultado corrobora o estudo de Ng, Debnath e Heesch (2017), no qual os autores identificaram que ciclistas tendem a preferir interseções em que o direito de passagem é dado aos motoristas e, com isso, podem decidir o melhor momento de cruzar a região mais crítica de conflitos dentro dos cruzamentos.

Posteriormente, foi realizado um ranqueamento das respostas de percepção de segurança para os 14 cenários avaliados pelo estudo, Tabela 1 . 0 ranqueamento foi realizado pelas medianas das respostas e, para os casos de cenários com o mesmo valor de mediana, os valores de moda e de média foram adotados como índice para desempate. Assim, como já observado anteriormente, é possível notar uma tendência de classificação dos cenários com cruzamentos entre vias compartilhadas como mais inseguros e cruzamentos entre vias com a presença de ciclovia no canteiro central como mais seguros.

Tabela 1 - Ranqueamento das respostas por cenário avaliado

\begin{tabular}{lllll}
\hline Posição & Cenário & Mediana & Moda & Média \\
\hline $1^{0}$ & Compartilhado - conflito de mesma direção com conversão à direita & 2 & 1 & 2,16 \\
$2^{0}$ & Compartilhado - conflito de cruzamento com aproximação pela esquerda & 2 & 1 & 2,32 \\
$3^{0}$ & Compartilhado - conflito direção oposta com conversão à esquerda & 2 & 2 & 2,31 \\
$4^{0}$ & Compartilhado - conflito de conversão à esquerda com aproximação pela direita & 2 & 3 & 2,41 \\
$5^{0}$ & Compartilhado - conflito de conversão à direita com aproximação pela direita & 3 & 3 & 2,58 \\
$6^{0}$ & Ciclofaixa - conflito de mesma direção com conversão à direita & 3 & 3 & 2,65 \\
$7^{0}$ & Ciclofaixa - conflito de conversão à esquerda com aproximação pela direita & 3 & 3 & 2,89 \\
$8^{0}$ & Ciclofaixa - conflito direção oposta com conversão à esquerda & 3 & 3 & 2,90 \\
$9^{0}$ & Ciclofaixa - conflito de cruzamento com aproximação pela esquerda & 3 & 3 & 2,93 \\
$10^{0}$ & Ciclovia - conflito de mesma direção com conversão à direita & 3 & 3 & 2,95 \\
$11^{0}$ & Ciclofaixa - conflito de conversão à direita com aproximação pela direita & 3 & 4 & 2,99 \\
$12^{0}$ & Ciclovia - Conflito de conversão à esquerda com aproximação pela direita & 3 & 4 & 3,17 \\
$13^{0}$ & Ciclovia - Conflito de cruzamento com aproximação pela esquerda & 3 & 4 & 3,21 \\
$14^{0}$ & Ciclovia - Conflito direção oposta com conversão à esquerda & 3 & 4 & 3,26 \\
\hline
\end{tabular}

0 ranqueamento das respostas sobre a percepção de segurança mostrou que o conflito de mesma direção com conversão à direita em cruzamentos entre vias compartilhadas foi o considerado como o mais inseguro pelos respondentes. Esse conflito é aquele em que o motorista e o ciclista estão seguindo na mesma direção e o motorista realiza conversão à direita na frente do ciclista, para os cenários com ciclovia essa conversão do motorista ocorre à esquerda. Essa tendência de perceber esse tipo de conflito como o mais inseguro pode ser explicada pela ausência de contato visual do ciclista com o motorista se aproximando por trás, contato esse já evidenciado como importante por NACTO (2014). 
0 resultado citado acima corrobora o levantamento de acidentalidade realizado por Johnson et al. (2014), na Austrália. No estudo os autores identificaram esse mesmo conflito como o responsável pela maioria dos acidentes por eles avaliados. Assim, para cenários com o conflito em que o ciclista e o motorista possuem pouco contato visual, além de influenciar na percepção de segurança, se mostra como o conflito mais perigoso em termos do risco real, que é referente a ocorrência de acidentes.

\subsubsection{Comparação das Repostas por Significância Estatística}

Nesta etapa, a percepção de segurança de um mesmo conflito foi testada em relação aos 3 tipos de infraestruturas por meio do teste de Kruskal-Wallis e, posteriormente, os cenários (conflito e infraestrutura) foram comparados entre si, em pares, por meio do teste de Mann-Whitney.

Assim sendo, a aplicação do teste de Kruskal-Wallis comparou cada um dos cinco tipos de conflitos em relação a uma infraestrutura diferente. Como exemplo, foi comparado se o conflito de mesma direção com conversão à direita é percebido da mesma forma nos cruzamentos com infraestrutura compartilhada, com ciclofaixa e com ciclovia no canteiro central.

Os resultados para esses testes indicaram que a percepção de segurança de um conflito é estatisticamente diferente quando se troca o tipo de infraestrutura, todos os resultados apresentaram valores de $p$ menor que 0,05 . Assim sendo, um conflito é percebido de forma diferente (em termos de segurança cicloviária) quando se troca o tipo de infraestrutura.

Ressalta-se que o conflito de conversão à direita com aproximação pela direita não ocorre em cruzamentos com a presença de ciclovia no canteiro central. Portanto, excepcionalmente para esse conflito, o teste de Kruskal-Wallis foi substituído pelo teste de Mann-Whitney, uma vez que somente duas variáveis estavam sendo comparadas. Conforme observado para os demais tipos de conflitos, o valor de $p$ foi menor que 0,5 , indicando que o tipo de infraestrutura também influencia na percepção de segurança desse conflito.

Em seguida, foi comparado um determinado cenário, ou seja, um tipo de conflito em uma determinada infraestrutura, em relação a todos os outros 13 cenários. Assim, foi comparado nessa etapa, por exemplo, a percepção de segurança para um cenário de cruzamento de vias compartilhadas em que ocorre um conflito de mesma direção com conversão à direita, em pares, com todos as outras combinações de conflito e infraestrutura.

Para tal análise foi utilizado o teste de Mann-Whitney, e constatou-se que a maioria dos resultados teve valor de $p$ menor que 0,5. Entretanto, foi observado uma tendência dos conflitos serem percebidos sem diferenças estatisticamente significativas (valores de $p>0,05$ ), com exceção do conflito de mesma direção com conversão à direita, em cruzamentos com as infraestruturas ciclofaixa e ciclovia. Além disso, o conflito de mesma direção com conversão à direita em cruzamentos entre vias com ciclovia no canteiro central é percebido de forma semelhante (valores de $p>0,05$ ) em relação a todos os demais conflitos em cruzamentos entre vias com a presença de ciclofaixa, com exceção apenas desse mesmo conflito nos cruzamentos entre vias com ciclofaixa.

\section{CONSIDERAÇÕES FINAIS}

0 presente estudo buscou identificar a percepção do ciclista em relação à segurança viária em cruzamentos urbanos não semaforizados, considerando a presença de 3 tipos de infraestrutura cicloviária (compartilhada, ciclofaixa e ciclovia). A motivação inicial desta pesquisa foi a necessidade de uma investigação em âmbito nacional sobre a percepção de segurança de ciclistas 
quanto a infraestrutura em cruzamentos não semaforizados, visto que ainda são escassos os estudos abordando esse tema.

A metodologia utilizada no estudo foi baseada em levantamento de dados por questionário on-line e análise pela aplicação de métodos de estatística descritiva e inferencial. Foram analisadas um total de 458 respostas que foram coletadas por um questionário aplicado no segundo semestre de 2018.

Os resultados encontrados indicaram uma preferência dos ciclistas por infraestrutura que segregam a circulação das bicicletas dos fluxos de automóveis motorizados nas aproximações de cruzamentos. Os cenários com cruzamentos entre vias com a presença de ciclovia no canteiro central foram classificados como os mais seguros. Os cenários com cruzamentos entre vias compartilhadas foram classificados como os mais inseguros. Esse resultado indica que os usuários de bicicletas tendem a preferir situações em cruzamentos onde a decisão de cruzar uma interseção pode ser realizada a partir de um ponto totalmente segregado dos modos de transporte motorizados. Assim sendo, o ciclista pode tomar a decisão do melhor momento de passar por um cruzamento e interagir com o fluxo de tráfego dos modos motorizados.

Nas avaliações, foram investigados cenários que envolviam uma série de conflitos e foi possível observar que os cenários em que ocorriam conflitos de mesma direção com conversão à direita (à esquerda para os cenários de ciclovia no canteiro central) na frente dos ciclistas foram considerados como os mais inseguros. Esse resultado indica que os ciclistas tendem a perceber situações com pouco contato visual como as mais inseguras.

Em conclusão, o presente artigo identificou os cruzamentos entre vias compartilhadas como os mais inseguros na percepção dos ciclistas. Nesse mesmo contexto, a interação de conflito de tráfego considerada a mais insegura foi aquela em que o automóvel se aproxima por trás do ciclista e realiza conversão à direita (à esquerda para os cenários de ciclovia no canteiro central).

Como recomendação para trabalhos futuros, sugere-se que seja incluído a ciclovia junto ao bordo lateral da via principal nas análises. Dessa forma, poderá ser objeto de estudo a comparativa da percepção da segurança entre ciclovia e ciclofaixa nos bordos da pista principal em cruzamentos não semaforizados.

Por fim, o artigo contribuiu para o maior entendimento, bem como para o direcionamento de estudos na área segurança cicloviária, uma vez que ainda são poucos os estudos encontrados neste campo que tenham sido desenvolvidos em território brasileiro. Além disso, os resultados indicam a importância de implementação de infraestrutura cicloviária para que seja promovida a utilização de bicicleta como meio de transporte de forma segura e funcional.

\section{AGRADECIMENTOS}

O presente trabalho foi realizado com apoio da Coordenação de Aperfeiçoamento de Pessoal de Nível Superior - Brasil (CAPES) - Código de Financiamento 001.

\section{REFERÊNCIAS}

Brasil (2010). Departamento Nacional de Infraestrutura de Transportes. Diretoria Executiva. Instituto de Pesquisas Rodoviárias.

Chataway, E. S.; Kaplan, S.; Nielsen, T. A. S.; Prato, C. G. (2014). Safety perceptions and reported behavior related to cycling in mixed traffic: A comparison between Brisbane and Copenhagen. Transportation Research Part F, n. 23, p. 32-43. DOI: 10.1016/j.trf.2013.12.021

Chaurand, N.; Delhomme, P. (2013). Cyclists and drivers in road interactions: A comparison of perceived crash risk. Accident Analysis and Prevention, v. 50, p. 1176-1184. DOI: 10.1016/j.aap.2012.09.005 
Denver Public Works (2016). Bicycle crash analysis: understanding and reducing bicycle \& motor vehicle crashes. Denver, CO: Transportation and Mobility.

Elvik, R. (2009). An exploratory analysis of models for estimating the combined effects of road safety measures. Accident Analysis and Prevention, v. 41, p. 876-880. DOI: 10.1016/j.aap.2009.05.003

EMBARQ (2015). Cities Safer by Design: Guidance and Examples to Promote Traffic Safety Through Urban and Streets Design. World Resources Institute.

Ferraz, A. C.; Raia Jr., A.; Bezerra, B; Bastos, T.; Rodrigues, K. (2012). Segurança Viária, São Carlos: Suprema Gráfica e Editora.

FHWA - Federal Highway Administration (2006). Lesson 3: Pedestrian and Bicyclist Safety. McLean, VA: Turner-Fairbank Highway Research Center.

Heinen, E.; Van Wee, B.; Maat, K. (2010). Commuting by Bicycle: An Overview of the Literature. Transport Reviews, sicpi, v. 30, n. 1, p. 59-96. DOI: $10.1080 / 01441640903187001$

IPEA - Instituto De Pesquisas Econômicas Aplicadas (2015). Acidentes de trânsito nas rodovias federais brasileiras: caracterização, tendências e custos para a sociedade. Brasília: Livraria Ipea.

Isaksson-Hellman, I. (2012). A study of bicycle and passenger car collisions based on insurance claims data. In: 56th AAAM Annual Conference, 2012. Annals of Advances in Automotive Medicine, v. 56, p. 3-12.

Johnson, M.; Chong, D.; Carroll, J; Katz R.; Oxley, J.; Charlton, J. (2014). Naturalistic Cycling Study: Identifying Risk Factors for Cyclists in the Australian Capital Territory. Monash University: Accident Research Centre.

Kaplan, S.; Prato, C. G. (2013). Cyclist-Motorist Crash Patterns in Denmark: A Latent Class Clustering Approach. Traffic Injury Prevention, v. 14, n. 7, p. 725-733. DOI: 10.1080/15389588.2012.759654

Landis, B.; Vattikuti, V. R.; Ottenberg, R. M.; Petritsch, T. A. (2003). Intersection Level of Service for the Bicycle Through Movement. Transportation Research Record. v. 1828, p. 101-106. DOI: 10.3141/1828-12

Lusk, A. C.; Furth, P. G.; Morency, P.; Miranda-Moreno, L. F.; Willett, W. C.; Dennerlein, J. T. (2011). Risk of injury for bicycling on cycle tracks versus in the street. Injury Prevention, v. 17, p. 131-135. DOI: 10.1136/ip.2010.028696

Manton, R.; Rau, H.; Fahy, F.; Sheahan, J.; Clifford, E. (2016). Using mental mapping to unpack perceived cycling risk. Accident Analysis and Prevention, v. 88, p. 138-149. DOI: 10.1016/j.aap.2015.12.017

Menghini G.; Carrasco, N.; Schüssler, N.; Axhausen, K. W. (2010). Route choice of cyclists in Zurich. Transportation Research Part A, v. 44, p. 754-765. DOI: 10.1016/j.tra.2010.07.008

Minikel, E. (2012). Cyclist safety on bicycle boulevards and parallel arterial routes in Berkeley, California. Accident Analysis and Prevention, v. 45, p. 241-247. DOI: 10.1016/j.aap.2011.07.009

Moller, M.; Hels, T. (2008). Cyclists' perception of risk in roundabouts. Accident Analysis and Prevention, v. 40, n. 3, p. 10551062. DOI: 10.1016/j.aap.2007.10.013

NACTO (2014). Urban Bikeway Design Guide. 2 ed. Washington, DC: National Association of City Transportation Officials.

Ng, A.; Debnath, A. K.; Heesch, K. C. (2017). Cyclist' safety perceptions of cycling infrastructure at un-signalised intersections: Cross-sectional survey of Queensland cyclists. Journal of Transport \& Health. v. 6, p. 13-22. DOI: 10.1016/j.jth.2017.03.001

Nordback, K.; Marshall, W. E.; Janson, B. N. (2014). Bicyclist safety performance functions for a U.S. city. Accident Analysis and Prevention, n. 65, p. 114-122. DOI: 10.1016/j.aap.2013.12.016

O'Connor, J. P.; Brown, T. D. (2010). Riding with the sharks: Serious leisure cyclist's perceptions of sharing the road with motorists. Journal of Science and Medicine in Sport, v. 13, p. 53-58. DOI: 10.1016/j.jsams.2008.11.003

Rasanem, M.; Summala, H. (1998). Attention and expectation problems in bicycle-car collisions: An in-depth study. Accident Analysis and Prevention, v. 30, n. 5, p. 657-666.

Rosenberg, A. (2015). Uso de bicicletas no Brasil: Qual melhor modelo de incentivo?. Rosenberg Associados: Brasil.

Teschke, K.; Harris, A.; Reynolds, C. C. O.; Winters, M.; Babul, S.; Chipman, M.; Cusimano, M. D.; Brubacher, J. R.; Hunte, G.; Friedman, S. M.; Monro, M.; Shen, H.; Vernich, L.; Cripton, P. A (2012). Route Infrastructure and the Risk of Injuries to Bicyclists: A Case-Crossover Study. American Journal of Public Health. v. 102, n. 12, p. 2336-2343. DOI: 10.2105/AJPH.2012.300762

Wachtel, B. A.; Lewiston, D. (1994). Risk factors for bicycle-motor vehicle collisions at intersections. ITE Journal, v. 64, n. 9, p. 30-35.

Waiselfisz, J. J. (2013). Mapa da Violência 2013: Acidentes de Trânsito e Motocicletas. Rio de Janeiro: CEBELA.

Wang, Y.; Nihan, N. L. (2004). Estimating the risk of collisions between bicycles and motor vehicles at signalized intersections. Accident Analysis and Prevention, v. 36, n. 2, p. 313-321. DOI: 10.1016/S0001-4575(03)00009-5

Wei, F.; Lovegrove, G. (2013). An empirical tool to evaluate the safety of cyclists: Community based, macro-level collision prediction models using negative binomial regression. Accident Analysis and Prevention, v. 61, p. 129-137. DOI: 10.1016/j.aap.2012.05.018

Weigand, L. (2008). A Review of Literature: Intersection Treatments to Improve Bicycle Access and Safety. Portland: Center for Transportation Studies, Portland State University.

Wegman, F.; Zhang, F; Dijkstra, A. (2012). How to make more cycling good for road safety?. Accident Analysis and Prevention, v. 44, n. 1, p. 19-29. DOI: 10.1016/j.aap.2010.11.010

Welle, B.; Li, W.; Adriazola-Steil, C. A. (2016). What Makes Cities Safer by Design? A Review of Evidence and Research on Practices to Improve Traffic Safety Through Urban and Street Design. Transportation Research Board: 95th Annual Meeting Compendium of Papers.

WHO - World Health Organization (2018). Global status report on road safety 2018. Geneva: World Health Organization. 